Please quote as: Bretschneider, U.; Gierczak, M.; Sonnick, A. \& Leimeister, J. M. (2015): Auf der Jagd nach dem günstigsten Preis: Was beeinflusst die Kaufabsicht von Nutzern von Produkt- und Preisvergleichsseiten?. In: Marktplätze im Umbruch Digitale Strategien für Services im Mobilen Internet (ISBN: 978-3-662-43782-7). Hrsg./Editors: Linnhoff-Popien, C.; Zaddach, M. \& Grahl, A. Verlag/Publisher: Springer Vieweg, Wiesbaden. Erscheinungsjahr/Year: 2015. Seiten/Pages: 43-53. 


\title{
Auf der Jagd nach dem günstigsten Preis: Was beeinflusst die Kaufabsicht von Nutzern von Produkt- und Preisvergleichsseiten?
}

\author{
Ulrich Bretschneider, Michael Marcin Gierczak, Anna Sonnick \\ und Jan Marco Leimeister
}

\section{Zusammenfassung}

Produkt- und Preisvergleichsseiten erfreuen sich immer größerer Beliebtheit. Nach einer Untersuchung des IfD Allensbach nutzen 54\% aller deutschen Internetnutzer Produkt- und Preisvergleichsseiten (PPS), um sich online über Produkte und deren Preise zu informieren. Aufgrund des relativen Neuheitsgrades dieses Phänomens, gibt es bislang wenige wissenschaftliche Untersuchungen zu PPS. Deshalb stellen diese den zentralen Untersuchungsgegenstand des vorliegenden Beitrages dar. Es soll empirisch überprüft werden, welche Faktoren im Rahmen der Nutzung solcher Seiten einen signifikanten Einfluss auf die Kaufabsicht haben. Dafür wird ein entsprechendes Hypothesenmodell aus der Theorie abgeleitet und anschließend empirisch überprüft.

U. Bretschneider $(\bowtie) \cdot$ M. M. Gierczak · A. Sonnick · J. M. Leimeister

Universität Kassel, Wirtschaftsinformatik, Kassel, Deutschland

E-Mail: bretschneider@uni-kassel.de

M. M. Gierczak

E-Mail: michael.gierczak@uni-kassel.de

A. Sonnick

E-Mail: anna.sonnick@wi-kassel.de

J. M. Leimeister

Universität St. Gallen, Institut für Wirtschaftsinformatik, St. Gallen, Schweiz

E-Mail: leimeister@uni-kassel.de, E-Mail: janmarco.leimeister@unisg.ch

(C) Springer-Verlag Berlin Heidelberg 2015

C. Linnhoff-Popien et al. (Hrsg.), Marktplätze im Umbruch, Xpert.press, DOI 10.1007/978-3-662-43782-7_7 


\subsection{Einleitung}

Das Internet wird im Allgemeinen als verbraucherfreundlich beschrieben, da es Konsumenten beim Kauf eine immense Produktauswahl sowie eine erhebliche Menge an Produktinformationen bietet. Mit Hilfe des Internets sind Konsumenten in der Lage, Preise zu vergleichen, nach Coupons und Rabatten zu suchen, bestimmte Marken zu recherchieren und Produktbewertungen von anderen Verbrauchern zu lesen. Des Weiteren sind diese Informationen unabhängig von Zeit und Ort und lediglich mit einem Mausklick verfügbar [3, S. 259, 20, S. 286].

Nach einer Computer- und Technik-Analyse des IfD Allensbach nutzen 54 \% aller deutschen Internetnutzer Produkt- und Preisvergleichsseiten (PPS), um sich online über Produkte und deren Preise zu informieren. Damit sind PPS die beliebteste Informationsquelle, noch vor Online-Shops und Hersteller-Webseiten [10]. Die zunehmende Relevanz von PPS zeigte sich bereits 2005 durch den Kauf von Shopping.com, einer Preisvergleichsseite mit hochentwickelter Technologie, durch Ebay für 620 Mio. \$ [6, S. 11]. Andere EBusiness Marktführer wie Amazon und Yahoo haben ebenfalls Produktempfehlungen auf ihren Webseiten integriert und dem Nutzer zugänglich gemacht [4].

Aufgrund des relativen Neuheitsgrades dieses Phänomens gibt es bislang wenige wissenschaftliche Untersuchungen zu PPS. Aus diesem Grund stellen diese den zentralen Untersuchungsgegenstand des vorliegenden Beitrages dar. Es soll empirisch gemessen werden, welche Einflussfaktoren, die aus der Nutzung solcher Seiten resultieren, einen signifikanten Einfluss auf die Kaufabsicht der Nutzer haben. Dafür wird ein entsprechendes Hypothesenmodell aus der Theorie abgeleitet und anschließend empirisch überprüft. Basierend auf den Ergebnissen erfolgt sodann die Ableitung von Handlungsempfehlungen und Implikationen für Forschung und Praxis.

\subsection{Theoretisches Untersuchungsmodell}

Dem Aufbau des Forschungsmodells der vorliegenden Studie wurde ein modifiziertes Technology Acceptance Modell (TAM) zugrunde gelegt. Dieses Modell nach [5] erlaubt Aussagen darüber, warum Individuen Technologien nutzen oder nicht nutzen. Ergänzend zu den ,instrumental beliefs“ (wahrgenommener Nutzen und wahrgenommene einfache Bedienbarkeit), welche die Hauptdeterminanten des TAM in seiner ursprünglichen Form sind, integriert das Modell der vorliegenden Studie zusätzlich ,affective beliefs“ und „trusting beliefs“, um zu erklären, wie die individuelle Kaufabsicht zustande kommt. Der Aufbau des Strukturmodells orientiert sich dabei an [1], die in ihrer Arbeit den Einfluss von Online-Produktempfehlungen auf die Kaufabsicht und die Wiederbenutzungsabsicht von Nutzern von Internet-basierten Recommendations Agents (RA), die vergleichbar mit PPS sind, untersuchten. Da PPS ebenfalls den RAs im weiteren Sinne zuzuordnen sind, wurde das von [1] entwickelte Modell als geeignet bewertet, um in abgeänderter Form die Beeinflussung der Kaufabsicht durch PPS zu untersuchen. Die Beeinflussung soll in 
der vorliegenden Arbeit in Anlehnung an [1] ebenfalls anhand der Konsumentenwahrnehmungen (consumer beliefs) gemessen werden, die im Wesentlichen eine individuelle Bewertung von Produkt- und Preisvergleichsseiten abbilden. Die Einteilung der Konsumentenwahrnehmungen in instrumental, trusting und affective beliefs basiert auf der von [22, S. 140 f.] vorgenommenen Klassifikation von Konsumenteneinstellungen hinsichtlich Recommendation Agents.

Neben den zwei dominanten Messgrößen zur Untersuchung der Konsumentenwahrnehmung im Hinblick auf Informationssysteme, der Nutzerzufriedenheit und der Technologieakzeptanz, wird in der Literatur auch das Vertrauen als maßgeblicher Einflussfaktor benannt [21, S. 85, 7, S. 52 f.]. Eine entsprechende Grundstruktur des Forschungsmodells unter Einbezug dieser drei Dimensionen findet eine Parallele in der Arbeit von [23]. Sein Konzept der ,,motivational affordance“ besagt, dass bei der Entwicklung neuer Informationssysteme (IS) die unterschiedlichen Motivationsquellen berücksichtigt werden sollten. Durch die Nutzung und Wiederbenutzung der IS befriedigt der Nutzer diverse psychologische, kognitive, soziale und emotionale Bedürfnisse. Demnach bestimmen die Eigenschaften, die diese Bedürfnisse (in der Summe bilden sie die „motivational affordance“) befriedigen, ob, wie und wie oft ein System genutzt wird, und den Gedankengang fortsetzend, potentiell auch, ob eine Kaufentscheidung gefällt wird.

Im Folgenden werden die Konstrukte, welche die drei Konsumentenwahrnehmungen (instrumental, trusting und affective beliefs) abbilden, näher erläutert.

Das „Vertrauen“ wird als erste Determinante, bei der ein positiver Einfluss auf die Kaufabsicht vermutet wird, in das dieser Arbeit zugrunde liegende Modell mit aufgenommen. In Anlehnung an [22] und [12] ist das „Vertrauen“ definiert als ,an individual's beliefs in a recomendation agent's competence, benevolence, and integrity“ [19, S. 76]. Diese drei Dimensionen wurden ebenfalls in einer großen Zahl weiterer Studien zur Messung des Vertrauens herangezogen (siehe hierzu [12] und werden auch der vorliegenden Arbeit zugrunde gelegt. In dem wachsenden Bereich der IS Forschung hat sich das „Vertrauen" bereits als ein wichtiger Antezedent der Technologieakzeptanz etabliert. Ebenso zeigen empirische Studien aus den Bereichen des E-Commerce und der RAs, dass das Vertrauen die Nutzungsabsicht des Konsumenten entweder direkt oder indirekt (über den wahrgenommenen Nutzen oder die wahrgenommene einfache Bedienbarkeit) beeinflusst (siehe hierzu [14, 15]). Gemäß [19] ist das Vertrauen in RAs eine Erweiterung des interpersonellen Vertrauens, d. h. des Vertrauens in eine Person. Die ,theory of social responses to computers" [16] besagt, dass Nutzer IT-Systeme wie soziale Interakteure behandeln, Vertrauen bilden und im übertragenen Sinn Beziehungen zu ihnen aufbauen [1, S. 246, 19, S. 222]. Vor diesem Hintergrund begründet sich eine erste Relevanz zur Integration der Dimensionen des interpersonellen Vertrauens (Kompetenz, Wohlwollen, Integrität) im folgenden Strukturmodell. Gleichzeitig belegen verschiedene Studien zu RAs, dass ebenso von einem direkten Einfluss des Vertrauens auf die Kaufabsicht ausgegangen werden kann $[4,11]$. In der vorliegenden Arbeit soll deshalb insbesondere untersucht werden, ob in vergleichbarer Weise eine positive Wirkbeziehung zwischen dem Vertrauen in PPS und der Kaufabsicht besteht. 


\section{Hypothese H1: Das Vertrauen in PPS hat einen positiven Einfluss auf die Kaufabsicht.}

Als weitere Determinante ist der „wahrgenommene Nutzen“ ein Faktor, dem ein positiver Einfluss auf die Kaufabsicht unterstellt wird. Laut [5, S. 320] versteht man unter dem wahrgenommenen Nutzen (perceived usefulness) ,the degree to which a person believes that using a particular system would enhance his or her [.] performance". Im Sinne dieser Definition untersucht das Konstrukt „wahrgenommener Nutzen" in der vorliegenden Arbeit den pragmatischen Aspekt, inwieweit die Nutzer davon überzeugt sind, dass PPS ihre Informationssuche hinsichtlich des gewünschten Produkts verbessern. Die Relevanz des wahrgenommenen Nutzens für die Verhaltensabsicht ergibt sich ferner aus theoretischer Sicht auch aus dem in der IS Forschung stark etablierten Technologieakzeptanzmodell [5]. Auch die Ergebnisse empirischer Studien zu verschiedenen anderen RAs legen nahe, dass eine Wirkbeziehung zwischen dem wahrgenommenen Nutzen und der Kaufabsicht besteht. So belegen beispielsweise Arbeiten zu Mobile Shopping [11] und RAs [1, 4] einen signifikant positiven Einfluss des wahrgenommenen Nutzens von RA-Technologien auf die Kaufabsicht. Dementsprechend wird für PPS in Hypothese 2 ebenfalls ein positiver Einfluss des wahrgenommenen Nutzens auf die Kaufabsicht angenommen.

Hypothese H2: Der wahrgenommene Nutzen von PPS hat einen positiven Einfluss auf die Kaufabsicht.

Die affektive Qualität wird, wie weiter oben bereits dargestellt, als weiterer wichtiger Faktor, der die Kaufabsicht beeinflusst, gehandelt. Um die Bedeutung dieses Konstrukts zu umreißen, müssen zunächst der sogenannte „,core affect“" und die affektive Qualität definiert werden. „Core affect is a neurophysiological state that is consciously accessible as a simple nonreflective feeling that is an integral blend of hedonic/valence (pleasure/displeasure) value and arousal/activation value (sleepy/activated)“ [17, S. 147]. Das bedeutet, der core affect untersucht inwieweit die Nutzer emotional auf PPS reagieren, ob man sich generell gut oder schlecht während der PPS-Nutzung fühlt und inwieweit PPS den Nutzer aktivieren und fesseln können [24, S. 106]. Darauf aufbauend wird die affektive Qualität nach [17, S. 147] als ,the ability to cause a change in core affect" definiert. Während der core affect seinen Ursprung im Innern einer Person hat, ist die affektive Qualität auf einen äußeren Stimulus zurückzuführen. Die wahrgenommene affektive Qualität (perceived affective quality) ist demnach, an individual's perception of the ability of a stimulus to change his or her core affect" [24, S. 106, 17, S. 157]. Die wahrgenommene affektive Qualität wird in der Regel anhand der Dimensionen des core affect gemessen: der Wertigkeit (hedonic/valence) (gut/schlecht) und der Aktivierung (arousal/activation) (ermüdend/ aktivierend) [24, S. 106].

Im Zusammenhang mit der Untersuchung von Einflussfaktoren des Nutzungsverhaltens in Bezug auf Webseiten entwickelte Zhang et al. [23] ein neues Konstrukt: die wahrgenommene visuelle Attraktivität. Dieses Konstrukt konzentriert sich lediglich auf die Wertigkeit (valence). Trotzdem ist von einer engen Verbundenheit der beiden Konstrukte auszugehen, da die Dimension „Wertigkeit“ der affektiven Qualität ebenfalls stark auf die 
Bewertung struktureller und visueller Aspekte abzielt [23, S. 286]. Darüber hinaus wurde in einer Studie von [13] belegt, dass die hedonische Qualität (hedonic quality) von bildschirmbasierten Informationssystemen (screen-based information systems), die sich z. B. in Farben, Abbildungen oder Musik ausdrückt, einen signifikant positiven Einfluss auf das wahrgenommene Vergnügen sowie die Akzeptanz hat. Einen vergleichbaren Zusammenhang beschreiben [9] die einen direkten Einfluss der wahrgenommenen Ästhetik auf die Verhaltensabsicht aufzeigen konnten. Jüngste Forschungsergebnisse hinsichtlich Recommendation Agents belegen ebenso, dass sich die wahrgenommene affektive Qualität als Bewertungsvariable eignet und ein positiver Einfluss auf die Kaufabsicht nachgewiesen werden kann [1, 4, 11, 18]. Vor diesem Hintergrund wird von einem direkten Einfluss der wahrgenommenen affektiven Qualität von PPS auf die Kaufabsicht ausgegangen.

Hypothese H3: Die wahrgenommene affektive Qualität von PPS hat einen positiven Einfluss auf die Kaufabsicht.

Neben den Forschungshypothesen werden darüber hinaus zwei weitere, aus der Forschung bekannte Wirkbeziehungen als zu replizierende Hypothesen in das Modell mit aufgenommen (siehe hierzu [1, 15, 19]). Im Einzelnen ist es sowohl der positive Einfluss des Vertrauens in PPS als auch der positive Einfluss der wahrgenommenen affektiven Qualität auf den wahrgenommenen Nutzen von PPS.

\subsection{Datenerhebung}

Im Rahmen der vorliegenden Arbeit wurden alle Konstrukte mit Hilfe von reflektiven Messmodellen operationalisiert. Alle Konstrukte des für diese Arbeit relevanten Strukturmodells wurden im Sinne der Multi-Item-Messung mit mindestens 3 Items oder mehr gemessen. Lediglich das Konstrukt „Kaufabsicht“ wurde mit Hilfe einer Single-Item-Messung umgesetzt. Alle in dieser Arbeit verwendeten Indikatoren wurden aus der Literatur zu einschlägigen Forschungsansätzen übernommen und bezüglich der Formulierung an das Themengebiet von PPS angepasst.

Im Fall der vorliegenden Untersuchung wurde die Form einer schriftlichen computergestützen Befragung gewählt. Mithilfe eines vollstandardisierten Online-Fragebogens konnte die Beantwortung einzeln und anhand festgelegter Antwortmöglichkeiten vorgenommen werden. Mit der Online-Befragung wurde im Hinblick auf den Forschungsgegenstandes eine Fokussierung besonders internetaffiner Zielgruppen angestrebt.

Die Umfrage endete nach fünf Wochen mit einem vorläufigen Datenbestand von 325 komplett abgeschlossenen Teilnahmen. Danach wurde jeder einzelne Datensatz auf Konsistenz bzw. die Anzahl fehlender Daten (Missing Values) überprüft. Ursprung der vorhandenen Missing Values war die Antwortmöglichkeit „Keine Angabe“. Daher musste den Empfehlungen von [8, S. 51] folgend ein Teil der Datensätze wegen fehlender Datenqualität ausgeschlossen werden. Dies betraf Datensätze, die a) mehr als 10\% Missing Values 
aufwiesen (94) und bei denen b) mehr als 50\% Missing Values innerhalb eines Konstrukts auftraten (5). Nach der Eliminierung verblieben insgesamt 226 Datensätze.

\subsection{Datenauswertung}

Die Datenauswertung erfolgte mittels PLS. Auf Messmodellebene wurden die Faktorbzw. Konstruktreliabilität und die durchschnittlich erfasste Varianz (DEV) der reflektiv operationalisierten Konstrukte mit Hilfe des PLS-Algorithmus in SmartPLS berechnet. Es weisen fast alle Items eine Faktorladung größer 0,7 auf, d. h. jeder einzelne Indikator teilt mehr als $50 \%$ seiner Varianz mit dem ihm zugeordnete Konstrukt. Lediglich fünf Indikatoren erreichten den Grenzwert von 0,7 nicht. Ihre entsprechenden Faktorladungen liegen alle zwischen 0,6 und 0,7. Im vorliegenden Fall haben jedoch all diese Indikatoren eine entscheidende Bedeutung für das jeweilige Konstrukt und wurden deshalb nicht eliminiert. Zudem konnte durch ihre Eliminierung kein oder nur ein unwesentlicher Anstieg der Konstruktreliabilität erreicht werden. Ergänzend zu der Indikatorreliabilität wurde anhand der Konstrukt- bzw. Faktorreliabilität untersucht, wie gut das jeweilige reflektive Konstrukt durch die ihm zugeordneten Indikatoren gemessen wird. Alle Konstrukte wiesen einen Wert deutlich über dem geforderten Grenzwert von 0,6 auf. Die Ergebnisse der durchschnittlich erfassten Varianz (DEV) liegen ebenfalls über dem festgelegten Mindestwert von 0,5, sodass auch hier keine Eliminierung von Indikatoren notwendig ist. Mit den präsentierten Ergebnissen wurden die Mindestanforderungen an die Konstruktreliabilität und die DEV deutlich überschritten, womit zusammengefasst von einer mehr als ausreichend dokumentierten Konvergenzreliabilität als Ergebnis dieser Auswertungen ausgegangen werden darf.

Abschließend erfolgte zur Beurteilung der Messmodelle die Überprüfung der Diskriminanzvalidität. Ein geeignetes Verfahren hierfür ist, das Fornell-Larcker Kriterium. Alle nach dem Fornell-Larcker-Kriterium errechneten Werte erreichten das Mindestmaß, so dass von ausreichender Diskriminanzvalidität im vorliegenden Fall ausgegangen werden kann. Da das Fornell-Larcker-Kriterium die Diskriminanzvalidität lediglich auf Konstruktebene untersucht, wird zusätzlich die Diskriminanzvalidität auf Indikatorebene anhand der Kreuzladungen beurteilt [2, S. 321, 8, S. 423]. Auch diesbezüglich wurden alle Mindestwerte erfüllt.

Auf Strukturmodellebene wurden im Rahmen der Gütebeurteilung die Pfadkoeffizienten, die Effektstärke $\mathrm{f}^{2}$, das Bestimmtheitsmaß $\mathrm{R}^{2}$ und die Prognoserelevanz $\mathrm{Q}^{2}$ herangezogen. Hier konnten bzgl. aller genannten Gütekriterien gute bis mittel-gute Werte erzielt werden, so dass dem Strukturmodell ebenfalls eine gewisse Güte bescheinigt werden konnte. Die wichtigsten Werte sind in Abb. 7.1 des Forschungsmodells abgetragen, aus der auch die Ergebnisse der Hypothesenprüfung hervorgeht. 


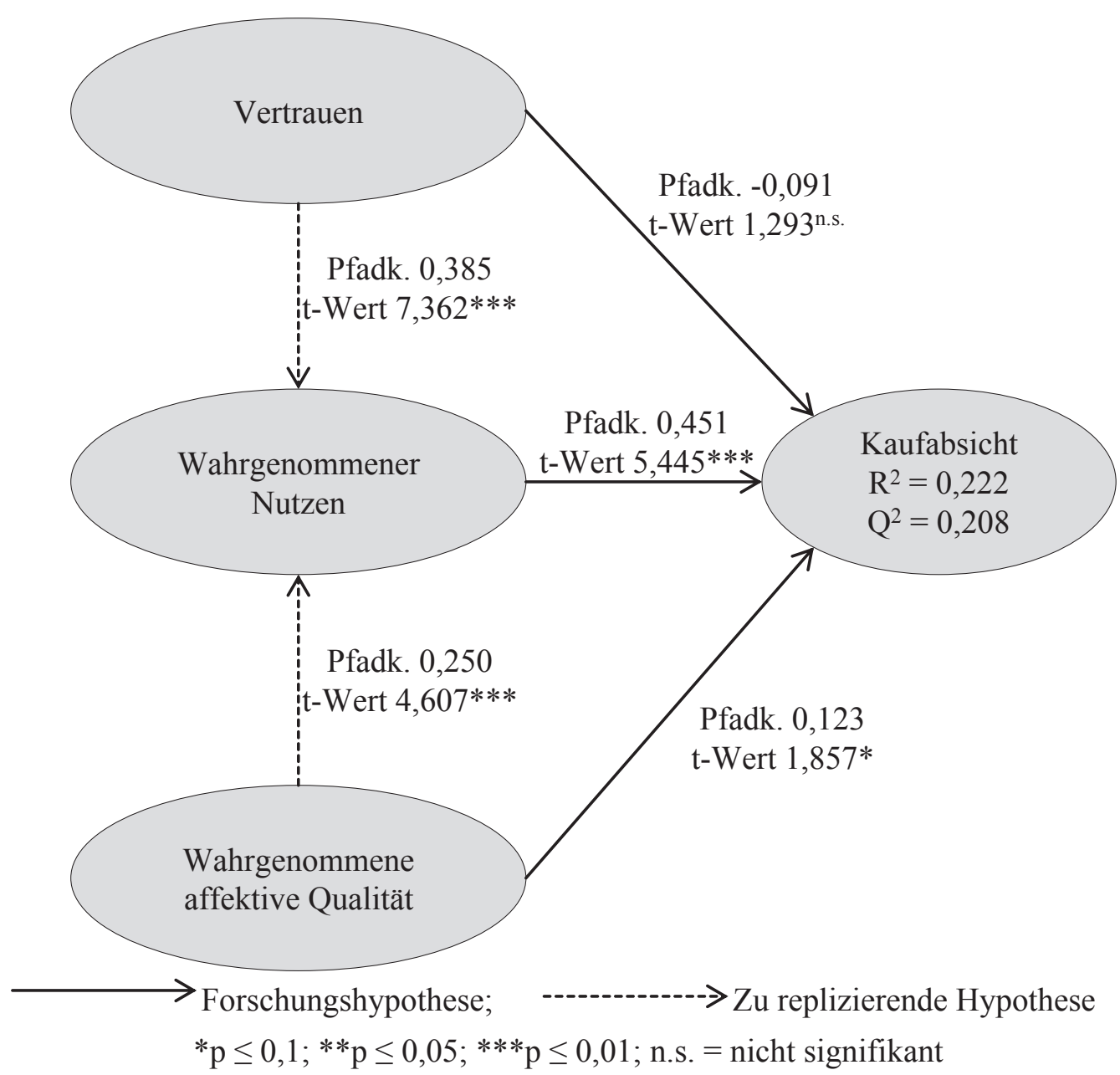

Abb. 7.1 Modell zum Einfluss von PPS auf die Kaufabsicht

\subsection{Diskussion}

In Bezug auf diese Kernfrage identifizieren die Ergebnisse des inneren Strukturmodells den „wahrgenommenen Nutzen“ als elementare Einflussgröße mit unmittelbarem Zusammenhang zur Kaufabsicht. Die Konsumentenwahrnehmungen „Vertrauen“ sowie „Wahrgenommene affektive Qualität“, basierend auf der Arbeit von [1], beeinflussen interessanterweise die unter Einbezug von PPS entstandene Kaufabsicht nicht als direkte Antezedenten. Dies steht im Kontrast zu den Studien von [1, 4, 11], in denen bezüglich anderer RAs ein direkter Einfluss der affektiven Qualität (bzw. Zufriedenheit) von RAs und des Vertrauens in RAs auf die Kaufabsicht nachgewiesen wurde. Hinsichtlich des wahrgenommenen Nutzens ergibt sich deutliche Kongruenz mit den Ergebnissen von [1], die sich mit der Auswirkung von Providerempfehlungen auf die Kaufabsicht befassten. Dieser übte dort ähnlich wie bei den hier untersuchten PPS einen stark signifikanten Einfluss auf die Kaufabsicht aus. Dies lässt als eines der zentralen Ergebnisse dieser Arbeit 
die Erkenntnis zu, dass bei der Nutzung von Produkt- und Preisvergleichsseiten vor allem die rational-instrumentellen Aspekte (im Sinne von „Nützlichkeit bei der Entscheidung“) für die Bildung einer Kaufabsicht vom Konsumenten als potentiell relevant erlebt werden. Diesen Zusammenhang bestätigen auch die Ergebnisse von [11], deren Arbeit ebenfalls darauf hinwies, dass nur die kognitive Ebene des Mobile Shoppings einen signifikanten Einfluss auf die Kaufabsicht aufweist.

Obwohl die Hypothese 3 aufgrund der Ergebnisse in Bezug auf die einzelnen zugehörigen Grenzwerte abgelehnt werden musste, soll an dieser Stelle doch noch einmal auf die schwach erkennbare Wirkbeziehung zwischen der wahrgenommenen affektiven Qualität und der Kaufabsicht eingegangen werden. In einem Versuch, diesen sich abzeichnenden Zusammenhang in einer erweiterten Sichtweise zu betrachten, kann man ihn in seiner inhaltlichen Aussage so verstehen, dass er darauf hindeutet, dass der affektive Reiz von PPS ein unspezifisches (Erregungs -) Potential im Sinne von ,arousal“ zur Aktivierung einer Kaufhandlung mobilisiert und damit die erlebte Kaufabsicht stärkt. Für die in dieser Arbeit erhobene Stichprobe konnte nur ein schwacher, signifikant grenzwertiger (10\%) Einfluss der affektiven Qualität belegt werden. Dennoch ist die ,wahrgenommene affektive Qualität" als potentiell beeinflussbare und damit zukünftig möglicherweise wachsende Einflussgröße zu betrachten. Wird dieser Aspekt in Zukunft z. B. von den Betreibern von PPS berücksichtigt und optimiert, könnte dies zu einer zunehmenden Bedeutung der Wirkbeziehung und einem erhöhten Einfluss der affektiven Qualität auf die Kaufabsicht führen.

Obwohl kein direkter Einfluss des Vertrauens und nur ein geringer und schwach signifikanter Einfluss der affektiven Qualität auf die Kaufabsicht besteht, konnte für beide zu replizierende Hypothesen ein signifikanter positiver Einfluss auf den wahrgenommenen Nutzen nachgewiesen werden, sodass zumindest von einem indirekten Einfluss des Vertrauens und der affektiven Qualität auf die Kaufabsicht ausgegangen werden kann, bei dem der über diese Konstrukte gesteigerte wahrgenommene Nutzen als Mediator agiert. Das bedeutet, Vertrauen und affektive Qualität tragen nicht direkt zur Bildung der Kaufabsicht bei, allerdings beeinflussen sie den wahrgenommenen Nutzen wesentlich. Je größer das Vertrauen der Nutzer in PPS ist, desto größer wird der Nutzen der von PPS ausgehenden Dienstleistungen erlebt. Von anderer Seite erhöht die affektive Qualität, also das emotionale Erlebnis mit bzw. die Attraktivität von PPS ebenfalls den erlebten Nutzen von PPS und somit auch die Kaufabsicht. Demnach können die beiden angesprochenen Wirkzusammenhänge für diese Studie als repliziert eingestuft werden.

\subsection{Implikationen}

Basierend auf den Ergebnissen und der Diskussion können praktische Implikationen für die Betreiber bzw. Designer von PPS abgeleitet werden.

Als zentrale Einflussgröße der aus der Nutzung von PPS resultierenden Kaufabsicht wurde der wahrgenommene Nutzen identifiziert. Demnach sollten für die Betreiber von 
PPS vor allem die Effektivität und Effizienz ihrer Seite im Mittelpunkt stehen. Hierzu können beispielsweise die Funktionalität, die Zuverlässigkeit bzw. Erreichbarkeit aber auch die Qualität der Informationen zählen. Betreiber sollten daher auf eine technische Stabilität der Seite achten (keine Ausfälle), den Informationsgehalt einer Produktempfehlung bzw. -anzeige möglichst gehaltvoll gestalten und Funktionalitäten von PPS weiter ausbauen bzw. neue Dienstleistungen integrieren. Durch die Optimierung dieser Punkte kann der wahrgenommene Nutzen und darüber auch die Kaufabsicht positiv beeinflusst werden.

Hinsichtlich des „Vertrauens“ in PPS ist vor allem die ihnen zugeschriebene Kompetenz als wichtiger Einflussfaktor zu betrachten. Dieser Aspekt beinhaltet unter anderem die Qualität der Produktinformation bzw. -empfehlung. Betreibern ist zu empfehlen, ihre Sachkunde hinsichtlich der zur Verfügung gestellten Inhalte in besonderer Weise erkennbar werden zu lassen. Welche Attribute hier besonders praxisrelevant werden, ist weiteren Untersuchungen vorbehalten. Neben der Kompetenz haben sowohl das „Wohlwollen“ als auch die „Integrität" von PPS wesentlichen Einfluss auf das Vertrauen und somit den wahrgenommenen Nutzen. Daher sollte bei der Image-Pflege von PPS diesen Aspekten besonders behutsame Berücksichtigung zukommen. Das bedeutet, Betreibern von PPS ist zu raten, eine neutrale, am Nutzer orientierte Stellung als intermediäres Medium zu wahren und z. B. keine Bevorzugung bestimmter Händler bzw. Produkte in ihre Empfehlungen zu integrieren. Sollte beim Nutzer der Verdacht entstehen, dass PPS keine objektiven Informationen anbieten, sondern neben Preis und Produktattributen noch andere, nicht im Interesse des Nutzers liegende Faktoren die PPS-Empfehlungen beeinflussen, könnte dies zu einer Senkung des wahrgenommenen Nutzens und somit zu einem negativen Einfluss auf die Kaufabsicht führen.

Auch von Seiten der ,,affektiven Qualität“ als grenzwertig signifikanter Einflussgröße der Kaufabsicht und direkter Einflussgröße des wahrgenommenen Nutzens lassen sich Implikationen ableiten. Um die affektive Qualität zu erhöhen und somit sowohl den wahrgenommen Nutzen als auch indirekt (und zu einem geringen Teil direkt) die Kaufabsicht positiv zu beeinflussen, ist den PPS-Betreibern zu empfehlen, neben der oben bereits besprochenen Konzentration auf die rational-instrumentelle Funktionalität auch das äußere Erscheinungsbild/Design der Seite möglichst ansprechend zu gestalten. PPS können aufgrund der Vielfalt an Dienstleistungen und Funktionen oft als unübersichtlich oder überladen empfunden werden. Daher erscheint es für PPS-Betreiber empfehlenswert, eine möglichst gut durchdachte visuelle Strukturiertheit der Seite zu gewährleisten. Zum Beispiel könnte man bei der Gestaltung von PPS Farben und Gliederung so einsetzen, dass sie ein möglichst langes Verweilen auf der Seite zulassen, ohne die Augen des Nutzers zu belasten. 


\section{Literatur}

1. Benlian, A., Titah, R., \& Hess, T. (2012). Differential effects of provider recommendations and consumer reviews in e-commerce transactions: An experimental study. Journal of Management Information Systems, 29(1), 237-272.

2. Chin, W. W. (1998b). The partial least squares approach for structural equation modeling. In G. A. Marcoulides (Hrsg.), Modern methods for business research (S. 295-336). Quantitative methodology series, Lawrence Erlbaum, Mahwah, New Jersey.

3. Dabholkar, P. A. (2006). Factors influencing consumer choice of a „rating web site“: An experimental investigation of an online interactive decision aid. Journal of Marketing Theory and Practice, 14(4), 259-273.

4. Dabhokar, P. A., \& Sheng, X. (2012). Consumer participation in using online recommendation agents: effects on satisfaction, trust, and purchase intentions. The Service Industries Journal, 32(9), 1433-1449.

5. Davis, F. D. (1989). Perceived usefulness, perceived ease of use, and user acceptance of information technology. MIS Quaterly, 13(3), 319-340.

6. Economist. (2005). Meg and the Power of Many- the world's biggest online auctioneer is trading on a new sort of future. Economist, 375(8429), 11.

7. Gefen, D., Karahanna, E., \& Straub, D. W. (2003). Trust and TAM in online shopping: An integrated model. MIS Quaterly, 27(1), 51-90.

8. Hair, J. F., Sarstedt, M., Ringle, C. M., \& Mena, J. A. (2012). An assessment of the use of partial least squares structural equation modeling in marketing research. Journal of the Academy of Marketing Science, 40, 414-433.

9. Hall, R. H., \& Hanna, P. (2004). The impact of web page text-background color combinations on readability, retention, aesthetics, and behavioral intention. Behavior \& Information Technology, 23(3), 183-195.

10. IfD Allensbach. (2011). Allensbacher Computer- und Technik-Analyse 2011. In: Statista, auf: http://de.statista.com/statistik/daten/studie/202544/umfrage/nutzung-von-recherchequellen-fuer-die-produktsuche-im-mobilen-internet/. Zugegriffen 1. Sept. 2014.

11. Kumar, A., \& Mukherjee, A. (2013). Shop while you talk: Determinants of purchase intentions through a mobile device. International Journal of Mobile Marketing, 8(1), 23-86.

12. McKnight, D.H., Choudhury, V., \& Kacmar, C. (2002). Developing and validating trust measures for e-commerce: An integrative typology. Information Systems Research, 13(3), 334-359.

13. Mundorf, N., Westin, S., \& Dholakia, N. (1993). Effects of hedonic components and user's gender on the acceptance of screen-based information services. Behaviour \& Information Technology, 12(5), 293-303.

14. Pavlou, P. A. (2003). Consumer acceptance of electronic commerce: Integrating trust and risk with the technology acceptance model. International Journal of Electronic Commerce, 7(3), 69-103.

15. Qiu, L., \& Benbasat, I. (2009). Evaluating anthropomorphic product recommendation agents: A social relationship perspective to designing information systems. Journal of Management Infomation Systems, 25(4), 145-181.

16. Reeves, B., \& Nass, C. (1996). The media equation: How people treat computers, television, and new media like real people and places. New York: Cambridge University Press.

17. Russell, J. A. (2003). Core affect and the psychological construction of emotion. Psychological Review, 110(1), 145-172.

18. Sanchez-Franco, M. J. (2010). WebCT - The quasimoderating effect of perceived affective quality on an extending technology acceptance model. Computers \& Education, 54, 37-46. 
19. Wang, W., \& Benbasat, I. (2005). Trust in and adoption of online recommendation agents. Journal of the Association for Information Systems, 6(3), 72-101.

20. West, P. M., Ariely, D., Bellman, S., Bradlow, E., Huber, J., Johnson, E., Kahn, B., Little, J., \& Schkade, D. (1999). Agents to the rescue? Marketing Letters, 10(3), 285-300.

21. Wixom, B. H., \& Todd, P. A. (2005). A theoretical integration of user satisfaction and technology acceptance. Information Systems Research, 16(1), 85-102.

22. Xiao, B., \& Benbasat, I. (2007). E-commerce product recommendation agents: Use, characteristics, and impact. MIS Quaterly, 31(1), 137-209.

23. Zhang, P., \& Li, N. (2004). Love at first sight or sustained effect? The role of perceived affective quality on users' cognitive reactions to IT. Proceedings of the International Conference on Information Systems (ICIS'04), Washington D.C.

24. Zhang P., \& Li, N. (2005). The importance of affective quality. Communications of the ACM, 48(9), 105-108. 


\title{
Auf der Jagd nach dem günstigsten Preis: Was beeinflusst die Kaufabsicht von Nutzern von Produkt- und Preisvergleichsseiten?
}

\author{
Ulrich Bretschneider, Michael Marcin Gierczak, Anna Sonnick \\ und Jan Marco Leimeister
}

\section{Zusammenfassung}

Produkt- und Preisvergleichsseiten erfreuen sich immer größerer Beliebtheit. Nach einer Untersuchung des IfD Allensbach nutzen 54\% aller deutschen Internetnutzer Produkt- und Preisvergleichsseiten (PPS), um sich online über Produkte und deren Preise zu informieren. Aufgrund des relativen Neuheitsgrades dieses Phänomens, gibt es bislang wenige wissenschaftliche Untersuchungen zu PPS. Deshalb stellen diese den zentralen Untersuchungsgegenstand des vorliegenden Beitrages dar. Es soll empirisch überprüft werden, welche Faktoren im Rahmen der Nutzung solcher Seiten einen signifikanten Einfluss auf die Kaufabsicht haben. Dafür wird ein entsprechendes Hypothesenmodell aus der Theorie abgeleitet und anschließend empirisch überprüft.

U. Bretschneider $(\bowtie) \cdot$ M. M. Gierczak · A. Sonnick · J. M. Leimeister

Universität Kassel, Wirtschaftsinformatik, Kassel, Deutschland

E-Mail: bretschneider@uni-kassel.de

M. M. Gierczak

E-Mail: michael.gierczak@uni-kassel.de

A. Sonnick

E-Mail: anna.sonnick@wi-kassel.de

J. M. Leimeister

Universität St. Gallen, Institut für Wirtschaftsinformatik, St. Gallen, Schweiz

E-Mail: leimeister@uni-kassel.de, E-Mail: janmarco.leimeister@unisg.ch

(C) Springer-Verlag Berlin Heidelberg 2015

C. Linnhoff-Popien et al. (Hrsg.), Marktplätze im Umbruch, Xpert.press, DOI 10.1007/978-3-662-43782-7_7 


\subsection{Einleitung}

Das Internet wird im Allgemeinen als verbraucherfreundlich beschrieben, da es Konsumenten beim Kauf eine immense Produktauswahl sowie eine erhebliche Menge an Produktinformationen bietet. Mit Hilfe des Internets sind Konsumenten in der Lage, Preise zu vergleichen, nach Coupons und Rabatten zu suchen, bestimmte Marken zu recherchieren und Produktbewertungen von anderen Verbrauchern zu lesen. Des Weiteren sind diese Informationen unabhängig von Zeit und Ort und lediglich mit einem Mausklick verfügbar [3, S. 259, 20, S. 286].

Nach einer Computer- und Technik-Analyse des IfD Allensbach nutzen 54 \% aller deutschen Internetnutzer Produkt- und Preisvergleichsseiten (PPS), um sich online über Produkte und deren Preise zu informieren. Damit sind PPS die beliebteste Informationsquelle, noch vor Online-Shops und Hersteller-Webseiten [10]. Die zunehmende Relevanz von PPS zeigte sich bereits 2005 durch den Kauf von Shopping.com, einer Preisvergleichsseite mit hochentwickelter Technologie, durch Ebay für 620 Mio. \$ [6, S. 11]. Andere EBusiness Marktführer wie Amazon und Yahoo haben ebenfalls Produktempfehlungen auf ihren Webseiten integriert und dem Nutzer zugänglich gemacht [4].

Aufgrund des relativen Neuheitsgrades dieses Phänomens gibt es bislang wenige wissenschaftliche Untersuchungen zu PPS. Aus diesem Grund stellen diese den zentralen Untersuchungsgegenstand des vorliegenden Beitrages dar. Es soll empirisch gemessen werden, welche Einflussfaktoren, die aus der Nutzung solcher Seiten resultieren, einen signifikanten Einfluss auf die Kaufabsicht der Nutzer haben. Dafür wird ein entsprechendes Hypothesenmodell aus der Theorie abgeleitet und anschließend empirisch überprüft. Basierend auf den Ergebnissen erfolgt sodann die Ableitung von Handlungsempfehlungen und Implikationen für Forschung und Praxis.

\subsection{Theoretisches Untersuchungsmodell}

Dem Aufbau des Forschungsmodells der vorliegenden Studie wurde ein modifiziertes Technology Acceptance Modell (TAM) zugrunde gelegt. Dieses Modell nach [5] erlaubt Aussagen darüber, warum Individuen Technologien nutzen oder nicht nutzen. Ergänzend zu den ,instrumental beliefs“ (wahrgenommener Nutzen und wahrgenommene einfache Bedienbarkeit), welche die Hauptdeterminanten des TAM in seiner ursprünglichen Form sind, integriert das Modell der vorliegenden Studie zusätzlich ,affective beliefs“ und „trusting beliefs“, um zu erklären, wie die individuelle Kaufabsicht zustande kommt. Der Aufbau des Strukturmodells orientiert sich dabei an [1], die in ihrer Arbeit den Einfluss von Online-Produktempfehlungen auf die Kaufabsicht und die Wiederbenutzungsabsicht von Nutzern von Internet-basierten Recommendations Agents (RA), die vergleichbar mit PPS sind, untersuchten. Da PPS ebenfalls den RAs im weiteren Sinne zuzuordnen sind, wurde das von [1] entwickelte Modell als geeignet bewertet, um in abgeänderter Form die Beeinflussung der Kaufabsicht durch PPS zu untersuchen. Die Beeinflussung soll in 
der vorliegenden Arbeit in Anlehnung an [1] ebenfalls anhand der Konsumentenwahrnehmungen (consumer beliefs) gemessen werden, die im Wesentlichen eine individuelle Bewertung von Produkt- und Preisvergleichsseiten abbilden. Die Einteilung der Konsumentenwahrnehmungen in instrumental, trusting und affective beliefs basiert auf der von [22, S. 140 f.] vorgenommenen Klassifikation von Konsumenteneinstellungen hinsichtlich Recommendation Agents.

Neben den zwei dominanten Messgrößen zur Untersuchung der Konsumentenwahrnehmung im Hinblick auf Informationssysteme, der Nutzerzufriedenheit und der Technologieakzeptanz, wird in der Literatur auch das Vertrauen als maßgeblicher Einflussfaktor benannt [21, S. 85, 7, S. 52 f.]. Eine entsprechende Grundstruktur des Forschungsmodells unter Einbezug dieser drei Dimensionen findet eine Parallele in der Arbeit von [23]. Sein Konzept der ,,motivational affordance“ besagt, dass bei der Entwicklung neuer Informationssysteme (IS) die unterschiedlichen Motivationsquellen berücksichtigt werden sollten. Durch die Nutzung und Wiederbenutzung der IS befriedigt der Nutzer diverse psychologische, kognitive, soziale und emotionale Bedürfnisse. Demnach bestimmen die Eigenschaften, die diese Bedürfnisse (in der Summe bilden sie die „motivational affordance“) befriedigen, ob, wie und wie oft ein System genutzt wird, und den Gedankengang fortsetzend, potentiell auch, ob eine Kaufentscheidung gefällt wird.

Im Folgenden werden die Konstrukte, welche die drei Konsumentenwahrnehmungen (instrumental, trusting und affective beliefs) abbilden, näher erläutert.

Das „Vertrauen“ wird als erste Determinante, bei der ein positiver Einfluss auf die Kaufabsicht vermutet wird, in das dieser Arbeit zugrunde liegende Modell mit aufgenommen. In Anlehnung an [22] und [12] ist das „Vertrauen“ definiert als ,an individual's beliefs in a recomendation agent's competence, benevolence, and integrity“ [19, S. 76]. Diese drei Dimensionen wurden ebenfalls in einer großen Zahl weiterer Studien zur Messung des Vertrauens herangezogen (siehe hierzu [12] und werden auch der vorliegenden Arbeit zugrunde gelegt. In dem wachsenden Bereich der IS Forschung hat sich das „Vertrauen" bereits als ein wichtiger Antezedent der Technologieakzeptanz etabliert. Ebenso zeigen empirische Studien aus den Bereichen des E-Commerce und der RAs, dass das Vertrauen die Nutzungsabsicht des Konsumenten entweder direkt oder indirekt (über den wahrgenommenen Nutzen oder die wahrgenommene einfache Bedienbarkeit) beeinflusst (siehe hierzu [14, 15]). Gemäß [19] ist das Vertrauen in RAs eine Erweiterung des interpersonellen Vertrauens, d. h. des Vertrauens in eine Person. Die ,theory of social responses to computers" [16] besagt, dass Nutzer IT-Systeme wie soziale Interakteure behandeln, Vertrauen bilden und im übertragenen Sinn Beziehungen zu ihnen aufbauen [1, S. 246, 19, S. 222]. Vor diesem Hintergrund begründet sich eine erste Relevanz zur Integration der Dimensionen des interpersonellen Vertrauens (Kompetenz, Wohlwollen, Integrität) im folgenden Strukturmodell. Gleichzeitig belegen verschiedene Studien zu RAs, dass ebenso von einem direkten Einfluss des Vertrauens auf die Kaufabsicht ausgegangen werden kann $[4,11]$. In der vorliegenden Arbeit soll deshalb insbesondere untersucht werden, ob in vergleichbarer Weise eine positive Wirkbeziehung zwischen dem Vertrauen in PPS und der Kaufabsicht besteht. 


\section{Hypothese H1: Das Vertrauen in PPS hat einen positiven Einfluss auf die Kaufabsicht.}

Als weitere Determinante ist der „wahrgenommene Nutzen“ ein Faktor, dem ein positiver Einfluss auf die Kaufabsicht unterstellt wird. Laut [5, S. 320] versteht man unter dem wahrgenommenen Nutzen (perceived usefulness) ,the degree to which a person believes that using a particular system would enhance his or her [.] performance". Im Sinne dieser Definition untersucht das Konstrukt „wahrgenommener Nutzen" in der vorliegenden Arbeit den pragmatischen Aspekt, inwieweit die Nutzer davon überzeugt sind, dass PPS ihre Informationssuche hinsichtlich des gewünschten Produkts verbessern. Die Relevanz des wahrgenommenen Nutzens für die Verhaltensabsicht ergibt sich ferner aus theoretischer Sicht auch aus dem in der IS Forschung stark etablierten Technologieakzeptanzmodell [5]. Auch die Ergebnisse empirischer Studien zu verschiedenen anderen RAs legen nahe, dass eine Wirkbeziehung zwischen dem wahrgenommenen Nutzen und der Kaufabsicht besteht. So belegen beispielsweise Arbeiten zu Mobile Shopping [11] und RAs [1, 4] einen signifikant positiven Einfluss des wahrgenommenen Nutzens von RA-Technologien auf die Kaufabsicht. Dementsprechend wird für PPS in Hypothese 2 ebenfalls ein positiver Einfluss des wahrgenommenen Nutzens auf die Kaufabsicht angenommen.

Hypothese H2: Der wahrgenommene Nutzen von PPS hat einen positiven Einfluss auf die Kaufabsicht.

Die affektive Qualität wird, wie weiter oben bereits dargestellt, als weiterer wichtiger Faktor, der die Kaufabsicht beeinflusst, gehandelt. Um die Bedeutung dieses Konstrukts zu umreißen, müssen zunächst der sogenannte „,core affect“" und die affektive Qualität definiert werden. „Core affect is a neurophysiological state that is consciously accessible as a simple nonreflective feeling that is an integral blend of hedonic/valence (pleasure/displeasure) value and arousal/activation value (sleepy/activated)“ [17, S. 147]. Das bedeutet, der core affect untersucht inwieweit die Nutzer emotional auf PPS reagieren, ob man sich generell gut oder schlecht während der PPS-Nutzung fühlt und inwieweit PPS den Nutzer aktivieren und fesseln können [24, S. 106]. Darauf aufbauend wird die affektive Qualität nach [17, S. 147] als ,the ability to cause a change in core affect" definiert. Während der core affect seinen Ursprung im Innern einer Person hat, ist die affektive Qualität auf einen äußeren Stimulus zurückzuführen. Die wahrgenommene affektive Qualität (perceived affective quality) ist demnach, an individual's perception of the ability of a stimulus to change his or her core affect" [24, S. 106, 17, S. 157]. Die wahrgenommene affektive Qualität wird in der Regel anhand der Dimensionen des core affect gemessen: der Wertigkeit (hedonic/valence) (gut/schlecht) und der Aktivierung (arousal/activation) (ermüdend/ aktivierend) [24, S. 106].

Im Zusammenhang mit der Untersuchung von Einflussfaktoren des Nutzungsverhaltens in Bezug auf Webseiten entwickelte Zhang et al. [23] ein neues Konstrukt: die wahrgenommene visuelle Attraktivität. Dieses Konstrukt konzentriert sich lediglich auf die Wertigkeit (valence). Trotzdem ist von einer engen Verbundenheit der beiden Konstrukte auszugehen, da die Dimension „Wertigkeit“ der affektiven Qualität ebenfalls stark auf die 
Bewertung struktureller und visueller Aspekte abzielt [23, S. 286]. Darüber hinaus wurde in einer Studie von [13] belegt, dass die hedonische Qualität (hedonic quality) von bildschirmbasierten Informationssystemen (screen-based information systems), die sich z. B. in Farben, Abbildungen oder Musik ausdrückt, einen signifikant positiven Einfluss auf das wahrgenommene Vergnügen sowie die Akzeptanz hat. Einen vergleichbaren Zusammenhang beschreiben [9] die einen direkten Einfluss der wahrgenommenen Ästhetik auf die Verhaltensabsicht aufzeigen konnten. Jüngste Forschungsergebnisse hinsichtlich Recommendation Agents belegen ebenso, dass sich die wahrgenommene affektive Qualität als Bewertungsvariable eignet und ein positiver Einfluss auf die Kaufabsicht nachgewiesen werden kann [1, 4, 11, 18]. Vor diesem Hintergrund wird von einem direkten Einfluss der wahrgenommenen affektiven Qualität von PPS auf die Kaufabsicht ausgegangen.

Hypothese H3: Die wahrgenommene affektive Qualität von PPS hat einen positiven Einfluss auf die Kaufabsicht.

Neben den Forschungshypothesen werden darüber hinaus zwei weitere, aus der Forschung bekannte Wirkbeziehungen als zu replizierende Hypothesen in das Modell mit aufgenommen (siehe hierzu [1, 15, 19]). Im Einzelnen ist es sowohl der positive Einfluss des Vertrauens in PPS als auch der positive Einfluss der wahrgenommenen affektiven Qualität auf den wahrgenommenen Nutzen von PPS.

\subsection{Datenerhebung}

Im Rahmen der vorliegenden Arbeit wurden alle Konstrukte mit Hilfe von reflektiven Messmodellen operationalisiert. Alle Konstrukte des für diese Arbeit relevanten Strukturmodells wurden im Sinne der Multi-Item-Messung mit mindestens 3 Items oder mehr gemessen. Lediglich das Konstrukt „Kaufabsicht“ wurde mit Hilfe einer Single-Item-Messung umgesetzt. Alle in dieser Arbeit verwendeten Indikatoren wurden aus der Literatur zu einschlägigen Forschungsansätzen übernommen und bezüglich der Formulierung an das Themengebiet von PPS angepasst.

Im Fall der vorliegenden Untersuchung wurde die Form einer schriftlichen computergestützen Befragung gewählt. Mithilfe eines vollstandardisierten Online-Fragebogens konnte die Beantwortung einzeln und anhand festgelegter Antwortmöglichkeiten vorgenommen werden. Mit der Online-Befragung wurde im Hinblick auf den Forschungsgegenstandes eine Fokussierung besonders internetaffiner Zielgruppen angestrebt.

Die Umfrage endete nach fünf Wochen mit einem vorläufigen Datenbestand von 325 komplett abgeschlossenen Teilnahmen. Danach wurde jeder einzelne Datensatz auf Konsistenz bzw. die Anzahl fehlender Daten (Missing Values) überprüft. Ursprung der vorhandenen Missing Values war die Antwortmöglichkeit „Keine Angabe“. Daher musste den Empfehlungen von [8, S. 51] folgend ein Teil der Datensätze wegen fehlender Datenqualität ausgeschlossen werden. Dies betraf Datensätze, die a) mehr als 10\% Missing Values 
aufwiesen (94) und bei denen b) mehr als 50\% Missing Values innerhalb eines Konstrukts auftraten (5). Nach der Eliminierung verblieben insgesamt 226 Datensätze.

\subsection{Datenauswertung}

Die Datenauswertung erfolgte mittels PLS. Auf Messmodellebene wurden die Faktorbzw. Konstruktreliabilität und die durchschnittlich erfasste Varianz (DEV) der reflektiv operationalisierten Konstrukte mit Hilfe des PLS-Algorithmus in SmartPLS berechnet. Es weisen fast alle Items eine Faktorladung größer 0,7 auf, d. h. jeder einzelne Indikator teilt mehr als $50 \%$ seiner Varianz mit dem ihm zugeordnete Konstrukt. Lediglich fünf Indikatoren erreichten den Grenzwert von 0,7 nicht. Ihre entsprechenden Faktorladungen liegen alle zwischen 0,6 und 0,7. Im vorliegenden Fall haben jedoch all diese Indikatoren eine entscheidende Bedeutung für das jeweilige Konstrukt und wurden deshalb nicht eliminiert. Zudem konnte durch ihre Eliminierung kein oder nur ein unwesentlicher Anstieg der Konstruktreliabilität erreicht werden. Ergänzend zu der Indikatorreliabilität wurde anhand der Konstrukt- bzw. Faktorreliabilität untersucht, wie gut das jeweilige reflektive Konstrukt durch die ihm zugeordneten Indikatoren gemessen wird. Alle Konstrukte wiesen einen Wert deutlich über dem geforderten Grenzwert von 0,6 auf. Die Ergebnisse der durchschnittlich erfassten Varianz (DEV) liegen ebenfalls über dem festgelegten Mindestwert von 0,5, sodass auch hier keine Eliminierung von Indikatoren notwendig ist. Mit den präsentierten Ergebnissen wurden die Mindestanforderungen an die Konstruktreliabilität und die DEV deutlich überschritten, womit zusammengefasst von einer mehr als ausreichend dokumentierten Konvergenzreliabilität als Ergebnis dieser Auswertungen ausgegangen werden darf.

Abschließend erfolgte zur Beurteilung der Messmodelle die Überprüfung der Diskriminanzvalidität. Ein geeignetes Verfahren hierfür ist, das Fornell-Larcker Kriterium. Alle nach dem Fornell-Larcker-Kriterium errechneten Werte erreichten das Mindestmaß, so dass von ausreichender Diskriminanzvalidität im vorliegenden Fall ausgegangen werden kann. Da das Fornell-Larcker-Kriterium die Diskriminanzvalidität lediglich auf Konstruktebene untersucht, wird zusätzlich die Diskriminanzvalidität auf Indikatorebene anhand der Kreuzladungen beurteilt [2, S. 321, 8, S. 423]. Auch diesbezüglich wurden alle Mindestwerte erfüllt.

Auf Strukturmodellebene wurden im Rahmen der Gütebeurteilung die Pfadkoeffizienten, die Effektstärke $\mathrm{f}^{2}$, das Bestimmtheitsmaß $\mathrm{R}^{2}$ und die Prognoserelevanz $\mathrm{Q}^{2}$ herangezogen. Hier konnten bzgl. aller genannten Gütekriterien gute bis mittel-gute Werte erzielt werden, so dass dem Strukturmodell ebenfalls eine gewisse Güte bescheinigt werden konnte. Die wichtigsten Werte sind in Abb. 7.1 des Forschungsmodells abgetragen, aus der auch die Ergebnisse der Hypothesenprüfung hervorgeht. 


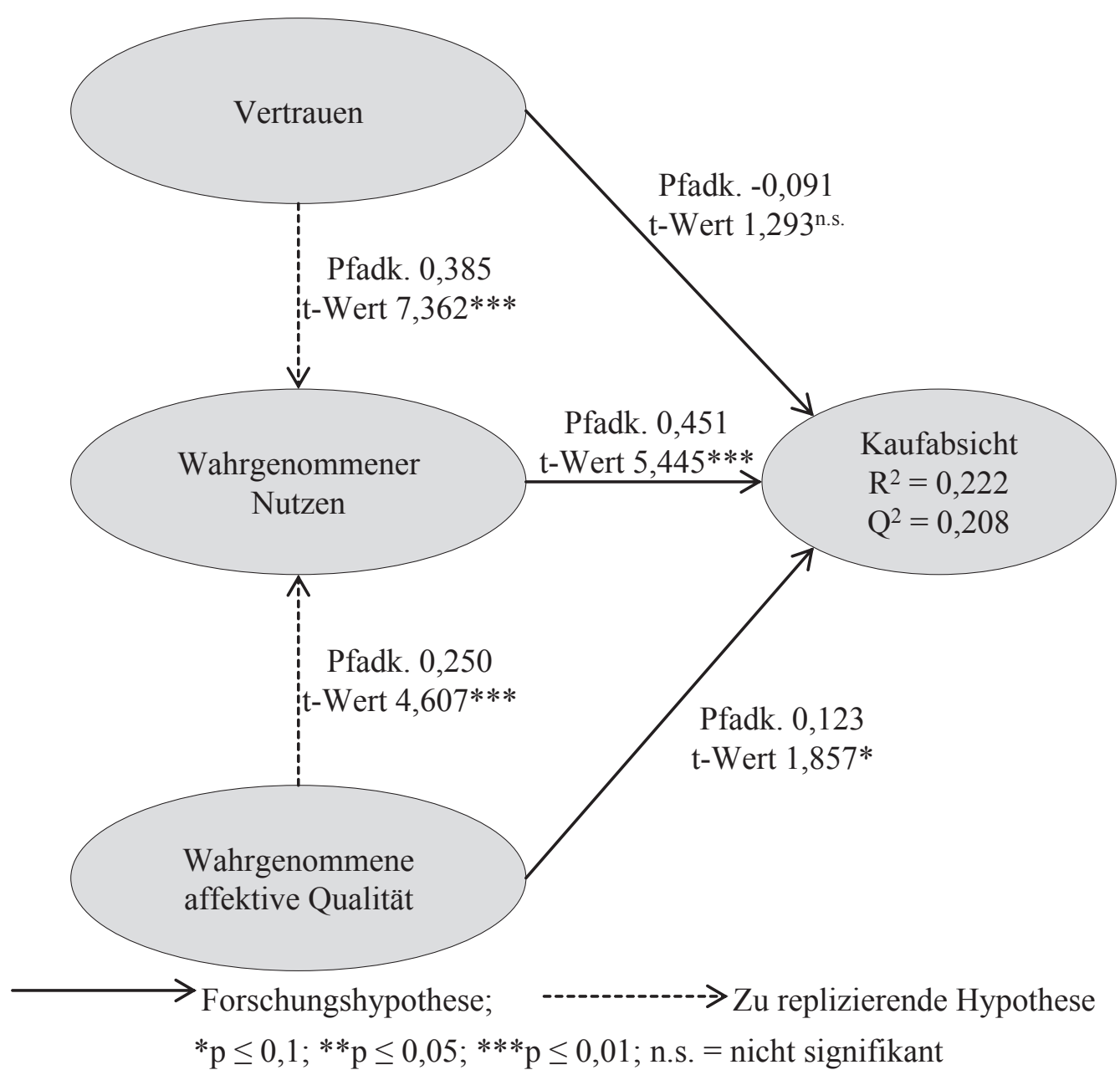

Abb. 7.1 Modell zum Einfluss von PPS auf die Kaufabsicht

\subsection{Diskussion}

In Bezug auf diese Kernfrage identifizieren die Ergebnisse des inneren Strukturmodells den „wahrgenommenen Nutzen“ als elementare Einflussgröße mit unmittelbarem Zusammenhang zur Kaufabsicht. Die Konsumentenwahrnehmungen „Vertrauen“ sowie „Wahrgenommene affektive Qualität“, basierend auf der Arbeit von [1], beeinflussen interessanterweise die unter Einbezug von PPS entstandene Kaufabsicht nicht als direkte Antezedenten. Dies steht im Kontrast zu den Studien von [1, 4, 11], in denen bezüglich anderer RAs ein direkter Einfluss der affektiven Qualität (bzw. Zufriedenheit) von RAs und des Vertrauens in RAs auf die Kaufabsicht nachgewiesen wurde. Hinsichtlich des wahrgenommenen Nutzens ergibt sich deutliche Kongruenz mit den Ergebnissen von [1], die sich mit der Auswirkung von Providerempfehlungen auf die Kaufabsicht befassten. Dieser übte dort ähnlich wie bei den hier untersuchten PPS einen stark signifikanten Einfluss auf die Kaufabsicht aus. Dies lässt als eines der zentralen Ergebnisse dieser Arbeit 
die Erkenntnis zu, dass bei der Nutzung von Produkt- und Preisvergleichsseiten vor allem die rational-instrumentellen Aspekte (im Sinne von „Nützlichkeit bei der Entscheidung“) für die Bildung einer Kaufabsicht vom Konsumenten als potentiell relevant erlebt werden. Diesen Zusammenhang bestätigen auch die Ergebnisse von [11], deren Arbeit ebenfalls darauf hinwies, dass nur die kognitive Ebene des Mobile Shoppings einen signifikanten Einfluss auf die Kaufabsicht aufweist.

Obwohl die Hypothese 3 aufgrund der Ergebnisse in Bezug auf die einzelnen zugehörigen Grenzwerte abgelehnt werden musste, soll an dieser Stelle doch noch einmal auf die schwach erkennbare Wirkbeziehung zwischen der wahrgenommenen affektiven Qualität und der Kaufabsicht eingegangen werden. In einem Versuch, diesen sich abzeichnenden Zusammenhang in einer erweiterten Sichtweise zu betrachten, kann man ihn in seiner inhaltlichen Aussage so verstehen, dass er darauf hindeutet, dass der affektive Reiz von PPS ein unspezifisches (Erregungs -) Potential im Sinne von ,arousal“ zur Aktivierung einer Kaufhandlung mobilisiert und damit die erlebte Kaufabsicht stärkt. Für die in dieser Arbeit erhobene Stichprobe konnte nur ein schwacher, signifikant grenzwertiger (10\%) Einfluss der affektiven Qualität belegt werden. Dennoch ist die ,wahrgenommene affektive Qualität" als potentiell beeinflussbare und damit zukünftig möglicherweise wachsende Einflussgröße zu betrachten. Wird dieser Aspekt in Zukunft z. B. von den Betreibern von PPS berücksichtigt und optimiert, könnte dies zu einer zunehmenden Bedeutung der Wirkbeziehung und einem erhöhten Einfluss der affektiven Qualität auf die Kaufabsicht führen.

Obwohl kein direkter Einfluss des Vertrauens und nur ein geringer und schwach signifikanter Einfluss der affektiven Qualität auf die Kaufabsicht besteht, konnte für beide zu replizierende Hypothesen ein signifikanter positiver Einfluss auf den wahrgenommenen Nutzen nachgewiesen werden, sodass zumindest von einem indirekten Einfluss des Vertrauens und der affektiven Qualität auf die Kaufabsicht ausgegangen werden kann, bei dem der über diese Konstrukte gesteigerte wahrgenommene Nutzen als Mediator agiert. Das bedeutet, Vertrauen und affektive Qualität tragen nicht direkt zur Bildung der Kaufabsicht bei, allerdings beeinflussen sie den wahrgenommenen Nutzen wesentlich. Je größer das Vertrauen der Nutzer in PPS ist, desto größer wird der Nutzen der von PPS ausgehenden Dienstleistungen erlebt. Von anderer Seite erhöht die affektive Qualität, also das emotionale Erlebnis mit bzw. die Attraktivität von PPS ebenfalls den erlebten Nutzen von PPS und somit auch die Kaufabsicht. Demnach können die beiden angesprochenen Wirkzusammenhänge für diese Studie als repliziert eingestuft werden.

\subsection{Implikationen}

Basierend auf den Ergebnissen und der Diskussion können praktische Implikationen für die Betreiber bzw. Designer von PPS abgeleitet werden.

Als zentrale Einflussgröße der aus der Nutzung von PPS resultierenden Kaufabsicht wurde der wahrgenommene Nutzen identifiziert. Demnach sollten für die Betreiber von 
PPS vor allem die Effektivität und Effizienz ihrer Seite im Mittelpunkt stehen. Hierzu können beispielsweise die Funktionalität, die Zuverlässigkeit bzw. Erreichbarkeit aber auch die Qualität der Informationen zählen. Betreiber sollten daher auf eine technische Stabilität der Seite achten (keine Ausfälle), den Informationsgehalt einer Produktempfehlung bzw. -anzeige möglichst gehaltvoll gestalten und Funktionalitäten von PPS weiter ausbauen bzw. neue Dienstleistungen integrieren. Durch die Optimierung dieser Punkte kann der wahrgenommene Nutzen und darüber auch die Kaufabsicht positiv beeinflusst werden.

Hinsichtlich des „Vertrauens“ in PPS ist vor allem die ihnen zugeschriebene Kompetenz als wichtiger Einflussfaktor zu betrachten. Dieser Aspekt beinhaltet unter anderem die Qualität der Produktinformation bzw. -empfehlung. Betreibern ist zu empfehlen, ihre Sachkunde hinsichtlich der zur Verfügung gestellten Inhalte in besonderer Weise erkennbar werden zu lassen. Welche Attribute hier besonders praxisrelevant werden, ist weiteren Untersuchungen vorbehalten. Neben der Kompetenz haben sowohl das „Wohlwollen“ als auch die „Integrität" von PPS wesentlichen Einfluss auf das Vertrauen und somit den wahrgenommenen Nutzen. Daher sollte bei der Image-Pflege von PPS diesen Aspekten besonders behutsame Berücksichtigung zukommen. Das bedeutet, Betreibern von PPS ist zu raten, eine neutrale, am Nutzer orientierte Stellung als intermediäres Medium zu wahren und z. B. keine Bevorzugung bestimmter Händler bzw. Produkte in ihre Empfehlungen zu integrieren. Sollte beim Nutzer der Verdacht entstehen, dass PPS keine objektiven Informationen anbieten, sondern neben Preis und Produktattributen noch andere, nicht im Interesse des Nutzers liegende Faktoren die PPS-Empfehlungen beeinflussen, könnte dies zu einer Senkung des wahrgenommenen Nutzens und somit zu einem negativen Einfluss auf die Kaufabsicht führen.

Auch von Seiten der ,,affektiven Qualität“ als grenzwertig signifikanter Einflussgröße der Kaufabsicht und direkter Einflussgröße des wahrgenommenen Nutzens lassen sich Implikationen ableiten. Um die affektive Qualität zu erhöhen und somit sowohl den wahrgenommen Nutzen als auch indirekt (und zu einem geringen Teil direkt) die Kaufabsicht positiv zu beeinflussen, ist den PPS-Betreibern zu empfehlen, neben der oben bereits besprochenen Konzentration auf die rational-instrumentelle Funktionalität auch das äußere Erscheinungsbild/Design der Seite möglichst ansprechend zu gestalten. PPS können aufgrund der Vielfalt an Dienstleistungen und Funktionen oft als unübersichtlich oder überladen empfunden werden. Daher erscheint es für PPS-Betreiber empfehlenswert, eine möglichst gut durchdachte visuelle Strukturiertheit der Seite zu gewährleisten. Zum Beispiel könnte man bei der Gestaltung von PPS Farben und Gliederung so einsetzen, dass sie ein möglichst langes Verweilen auf der Seite zulassen, ohne die Augen des Nutzers zu belasten. 


\section{Literatur}

1. Benlian, A., Titah, R., \& Hess, T. (2012). Differential effects of provider recommendations and consumer reviews in e-commerce transactions: An experimental study. Journal of Management Information Systems, 29(1), 237-272.

2. Chin, W. W. (1998b). The partial least squares approach for structural equation modeling. In G. A. Marcoulides (Hrsg.), Modern methods for business research (S. 295-336). Quantitative methodology series, Lawrence Erlbaum, Mahwah, New Jersey.

3. Dabholkar, P. A. (2006). Factors influencing consumer choice of a „rating web site“: An experimental investigation of an online interactive decision aid. Journal of Marketing Theory and Practice, 14(4), 259-273.

4. Dabhokar, P. A., \& Sheng, X. (2012). Consumer participation in using online recommendation agents: effects on satisfaction, trust, and purchase intentions. The Service Industries Journal, 32(9), 1433-1449.

5. Davis, F. D. (1989). Perceived usefulness, perceived ease of use, and user acceptance of information technology. MIS Quaterly, 13(3), 319-340.

6. Economist. (2005). Meg and the Power of Many- the world's biggest online auctioneer is trading on a new sort of future. Economist, 375(8429), 11.

7. Gefen, D., Karahanna, E., \& Straub, D. W. (2003). Trust and TAM in online shopping: An integrated model. MIS Quaterly, 27(1), 51-90.

8. Hair, J. F., Sarstedt, M., Ringle, C. M., \& Mena, J. A. (2012). An assessment of the use of partial least squares structural equation modeling in marketing research. Journal of the Academy of Marketing Science, 40, 414-433.

9. Hall, R. H., \& Hanna, P. (2004). The impact of web page text-background color combinations on readability, retention, aesthetics, and behavioral intention. Behavior \& Information Technology, 23(3), 183-195.

10. IfD Allensbach. (2011). Allensbacher Computer- und Technik-Analyse 2011. In: Statista, auf: http://de.statista.com/statistik/daten/studie/202544/umfrage/nutzung-von-recherchequellen-fuer-die-produktsuche-im-mobilen-internet/. Zugegriffen 1. Sept. 2014.

11. Kumar, A., \& Mukherjee, A. (2013). Shop while you talk: Determinants of purchase intentions through a mobile device. International Journal of Mobile Marketing, 8(1), 23-86.

12. McKnight, D.H., Choudhury, V., \& Kacmar, C. (2002). Developing and validating trust measures for e-commerce: An integrative typology. Information Systems Research, 13(3), 334-359.

13. Mundorf, N., Westin, S., \& Dholakia, N. (1993). Effects of hedonic components and user's gender on the acceptance of screen-based information services. Behaviour \& Information Technology, 12(5), 293-303.

14. Pavlou, P. A. (2003). Consumer acceptance of electronic commerce: Integrating trust and risk with the technology acceptance model. International Journal of Electronic Commerce, 7(3), 69-103.

15. Qiu, L., \& Benbasat, I. (2009). Evaluating anthropomorphic product recommendation agents: A social relationship perspective to designing information systems. Journal of Management Infomation Systems, 25(4), 145-181.

16. Reeves, B., \& Nass, C. (1996). The media equation: How people treat computers, television, and new media like real people and places. New York: Cambridge University Press.

17. Russell, J. A. (2003). Core affect and the psychological construction of emotion. Psychological Review, 110(1), 145-172.

18. Sanchez-Franco, M. J. (2010). WebCT - The quasimoderating effect of perceived affective quality on an extending technology acceptance model. Computers \& Education, 54, 37-46. 
19. Wang, W., \& Benbasat, I. (2005). Trust in and adoption of online recommendation agents. Journal of the Association for Information Systems, 6(3), 72-101.

20. West, P. M., Ariely, D., Bellman, S., Bradlow, E., Huber, J., Johnson, E., Kahn, B., Little, J., \& Schkade, D. (1999). Agents to the rescue? Marketing Letters, 10(3), 285-300.

21. Wixom, B. H., \& Todd, P. A. (2005). A theoretical integration of user satisfaction and technology acceptance. Information Systems Research, 16(1), 85-102.

22. Xiao, B., \& Benbasat, I. (2007). E-commerce product recommendation agents: Use, characteristics, and impact. MIS Quaterly, 31(1), 137-209.

23. Zhang, P., \& Li, N. (2004). Love at first sight or sustained effect? The role of perceived affective quality on users' cognitive reactions to IT. Proceedings of the International Conference on Information Systems (ICIS'04), Washington D.C.

24. Zhang P., \& Li, N. (2005). The importance of affective quality. Communications of the ACM, 48(9), 105-108. 